\title{
Chiral magnetic wave in an expanding QCD fluid
}

\author{
Seyed Farid Taghavi ${ }^{1,2, *}$ and Urs Achim Wiedemann ${ }^{2, \dagger}$ \\ ${ }^{1}$ School of Particles and Accelerators, Institute for Research in Fundamental Sciences (IPM), P.O. Box 19395-5531, Tehran, Iran \\ ${ }^{2}$ Physics Department, Theory Unit, CERN, CH-1211 Genève 23, Switzerland \\ (Received 9 March 2014; revised manuscript received 15 December 2014; published 4 February 2015)
}

\begin{abstract}
As a consequence of the chiral anomaly, the hydrodynamics of hot quantum chromodynamics (QCD) matter coupled to quantum electrodynamics allows for a long-wavelength mode of chiral charge density, the chiral magnetic wave (CMW), that provides for a mechanism of electric charge separation along the direction of an external magnetic field. Here, we investigate the efficiency of this mechanism for values of the time-dependent magnetic field and of the energy density attained in the hot QCD matter of ultrarelativistic heavy-ion collisions. To this end, we derive the CMW equations of motion for expanding systems by treating the CMW as a charge perturbation on top of an expanding Bjorken-type background field in the limit $\mu / T \ll 1$. Both, approximate analytical and full numerical solutions to these equations of motion, indicate that for the lifetime and thermodynamic conditions of ultrarelativistic heavy-ion collisions, the efficiency of CMW-induced electric charge separation decreases with increasing center-of-mass energy and that the effect is numerically very small. We note, however, that if sizable oriented asymmetries in the axial charge distribution (that are not induced by the CMW) are present in the early fluid dynamic evolution, then the mechanism of CMW-induced electric charge separation can be much more efficient.
\end{abstract}

DOI: 10.1103/PhysRevC.91.024902

PACS number(s): 12.38.Mh, 24.85.+p

\section{INTRODUCTION}

Our understanding of how the chiral anomaly affects the dynamics of relativistic plasmas has deepened considerably in recent years. The appearance of macroscopic parity-violating currents from quantum anomalies was noted already in the 1990s for systems as different as superfluid ${ }^{3} \mathrm{He}-\mathrm{A}[1,2]$, and the electroweak plasma in the early universe [3,4]. Without direct connection to the chiral anomaly, the possibility of parityviolating currents had been noted even earlier by Vilenkin for the case of neutrino-emission from a thermal system with chiral fermions that is either rotating $[5,6]$ or embedded in an external magnetic field [7]. As understood more recently, it is a direct consequence of the second law of thermodynamics that the viscous relativistic hydrodynamics of any charged plasma with triangle anomaly carries currents with terms proportional to the vorticity and proportional to an external magnetic field [8]. Rather than being set by new material properties, the strength of these anomalous contributions is given in terms of known thermodynamic functions and it is generally finite for nonvanishing chemical potentials. Anomalous hydrodynamics therefore must display currents with features that do not have a direct counterpart in plasmas without quantum anomalies. The charged plasmas of quantum field theories with gravity duals provide examples for which these features arise in explicit calculations [9-13]. There is also significant progress in understanding how anomalous hydrodynamics arises in the

\footnotetext{
*s.f.taghavi@ipm.ir

†urs.wiedemann@cern.ch
}

Published by the American Physical Society under the terms of the Creative Commons Attribution 3.0 License. Further distribution of this work must maintain attribution to the author(s) and the published article's title, journal citation, and DOI. long wavelength limit of classical kinetic theory supplemented by the notion of Berry curvature [14-16], how it emerges in the quantum kinetic approach $[17,18]$, and which terms arise in anomalous hydrodynamics beyond the first order in viscous corrections [19].

In the quark-gluon plasma of the phenomenologically realized quantum field theory of quantum chromodynamics (QCD) coupled to electrodynamics, the fermionic degrees of freedom carry both, the conserved electromagnetic and the anomalous axial charge. The collective close-to-equilibrium dynamics of the quark-gluon plasma provides therefore a special case of anomalous hydrodynamics [20]: For nonvanishing chemical potential of the axial charge $\mu_{A}$, an external magnetic field $\vec{B}$ or a vorticity $\vec{\omega}$ in the fluid induces an electromagnetic vector current $\vec{j}_{V}$. And for nonvanishing vector chemical potential $\mu_{V}$, vorticity or an external magnetic field induces an axial current $\vec{j}_{A}$.

The question to what extent these characteristic features of the chiral anomaly could lead to experimentally accessible signatures in the plasma produced in ultrarelativistic heavy-ion collisions is currently at the focus of an intense theoretical debate [21-33] and of experimental searches [34-42]. In this context, the mechanism of electric charge separation via the chiral magnetic wave (CMW) is of particular interest. The CMW was proposed first in Ref. [28] and then applied to the case of finite baryon density in Refs. [30,31]. It is a long-wavelength mode of chiral charge density that is an unavoidable consequence of the chiral anomaly in QCD hydrodynamics coupled to quantum electrodynamics. The CMW follows from general principles without any model-dependent assumption (as can be seen, e.g., from our generalization of the CMW to expanding fluids in Sec. II below). Thus, there is a controlled model-independent dynamical framework, namely anomalous hydrodynamics, within which one can discuss the efficiency of mechanisms of electric charge separation in 
heavy-ion collisions. ${ }^{1}$ The main purpose of the present work is to contribute to this discussion with a study of the CMW that accounts for major confounding factors in ultrarelativistic heavy-ion collisions, such as the strong time dependence of the energy and charge densities attained in the collisions and the transient nature of the generated magnetic field.

Our work is organized as follows: In Sec. II, we derive the equations of motion of the chiral magnetic wave as a charge perturbation for the simplified case of an expanding Bjorken-type model. We explain how this leads to a transparent model in which the efficiency of electric charge separation in response to an external magnetic field can be studied analytically and numerically. After discussing in Sec. III A an approximate analytical solution of the chiral magnetic wave that reveals already the main features of the full solution, we turn in Secs. III B and IV to a numerical study of how efficient the chiral magnetic wave is in separating electric charges. We discuss how our results are related to (and consistent with) previous discussions of the chiral magnetic wave, and we discuss conclusions.

\section{ANOMALOUS HYDRODYNAMICS}

We consider a model of the energy-momentum tensor $T^{\mu \nu}$ of hot QCD matter in an external electromagnetic field $F^{\mu \nu}$. This matter carries both electric charge (written here in terms of the vector current $j_{V}^{\mu}=\bar{\psi} \gamma^{\mu} \psi$ ) and axial charge (with axial current $\left.j_{A}^{\mu}=\bar{\psi} \gamma^{\mu} \gamma^{5} \psi\right)$. The electromagnetic field couples to the vector current, and it determines the divergence of the anomalous axial current. The fluid dynamic equations of motion of this system read

$$
\begin{aligned}
\nabla_{\mu} T^{\mu \nu} & =Q_{V} F^{v \lambda} j_{V \lambda}, \\
\nabla_{\mu} j_{V}^{\mu} & =0 \\
\nabla_{\mu} j_{A}^{\mu} & =C E . B
\end{aligned}
$$

where the external electric and magnetic field is defined via a tensor decomposition of $F^{\mu \nu}$ with respect to the flow field $u^{\mu}, E^{\mu}=F^{\mu v} u_{v}$, and $B^{\mu}=\frac{1}{2} \epsilon^{\mu \nu \alpha \beta} u^{v} F_{\alpha \beta}$. We use a convention with $j_{\text {electric }}^{\mu}=e j_{V}^{\mu}$, and where one power of $e$ is absorbed in the definition of $j_{A}^{\mu}$, accordingly. The strength of the Abelian anomaly is then $C=e / 2 \pi^{2}$. For simplicity, we set the vector charge to $Q_{V}=e$. This neglects that different quark flavors carry different fractional electric charge. (To avoid this simplification, one would have to introduce flavor-dependent currents and chemical potentials.) It will

\footnotetext{
${ }^{1}$ The caveat here is that the application of fluid dynamics is limited to sufficiently late times when gradients are small, while the effect of the CMW arises at sufficiently early times when the magnetic field is large. In the numerical studies of Secs. III and IV, we shall typically work with initialization times $\tau_{0}=0.1 \mathrm{fm} / \mathrm{c}$. We regard the extrapolation of hydrodynamics to these early times as the best possible dynamical formulation of the CMW available at present. Given that the main conclusions of Secs. III and IV depend only on the logarithmic order of magnitude of charge separation, there is an a posteriori argument that the inclusion of nonequilibrium effects at early times should not affect these conclusions significantly.
}

be sometimes convenient to work in the basis of left- and right-handed currents,

$$
j_{R}^{\mu}=\frac{1}{2}\left(j_{V}^{\mu}+j_{A}^{\mu}\right) \quad j_{L}^{\mu}=\frac{1}{2}\left(j_{V}^{\mu}-j_{A}^{\mu}\right) .
$$

Requiring that the second law of thermodynamics is satisfied locally, i.e., that the divergence of the entropy current is positive semidefinite everywhere, one can write Eq. (1) explicitly in the gradient expansion of viscous fluid dynamics. Following Son and Surowka [8], the energy momentum tensor and currents read to first order [20]:

$$
\begin{aligned}
& T^{\mu \nu}=(\epsilon+P) u^{\mu} u^{\nu}+P g^{\mu \nu}+\tau^{\mu \nu}, \\
& j_{R, L}^{\mu}=n_{R, L} u^{\mu}+v_{R, L}^{\mu},
\end{aligned}
$$

where

$$
\begin{aligned}
\tau^{\mu \nu}= & -\eta_{s} \Delta^{\mu \alpha} \Delta^{\nu \beta}\left(\nabla_{\alpha} u_{\beta}+\nabla_{\beta} u_{\alpha}\right) \\
& -\left(\zeta-\frac{2}{3} \eta_{s}\right) \Delta^{\mu \nu} \nabla \cdot u, \\
\nu_{R, L}^{\mu}= & -\frac{\sigma}{2}\left(T \Delta^{\mu \nu} \partial_{\nu}\left(\frac{\mu_{R, L}}{T}\right)+E^{\mu}\right) \\
& +\xi_{R, L} \omega^{\mu}+\xi_{R, L}^{(B)} B^{\mu} .
\end{aligned}
$$

Here, the nonideal part $\tau^{\mu \nu}$ of the energy-momentum tensor is characterized by the shear viscous transport coefficient $\eta_{s}$ and the bulk viscous coefficient $\zeta$. Up to first order, $\tau^{\mu \nu}$ can be written in terms of gradients of the flow field $u^{\mu}$ and the projector $\Delta^{\mu \nu}=g^{\mu \nu}+u^{\mu} u^{\nu}$. The viscous part $v_{R, L}^{\mu}$ of the left- and right-handed currents $j_{R, L}^{\mu}$ contains a conventional term that is proportional to the vector charge conductivity $\sigma$. This contribution describes how the currents flow along an electric field or a gradient in the chemical potentials. The contribution of the external electric field in (6) is negligible, of course, if the electric field is much smaller than the magnetic one. In addition, if the external electric field is sizable but its orientation differs from that of the magnetic field, this may allow one to disentangle the standard charge separation because of the ohmic contribution $\propto \sigma E^{\mu}$ from the anomaly-induced charge separation along the direction of an external magnetic field. In recent works that discuss the efficiency of the anomaly-induced charge separation effect, it is therefore not uncommon to neglect this ohmic contribution, and to focus on the terms in the second line of (6) that render the hydrodynamics of the system (1) anomalous [30,31]. We shall follow this approach. We note, however, that there are models of initial conditions for which $\vec{E}$ and $\vec{B}$ have similar orientation $[33,43]$. In this case, the Ohmic contribution in (6) is clearly relevant for describing charge transport in the direction of the magnetic field. By neglecting this contribution in the following, we shall quantify then not the total charge density floating along $\vec{B}$, but only the contribution to this charge density flow induced by the anomalous part of the current. It is in this sense that our paper does not provide a full phenomenological study but only the quantification of a novel effect relevant for phenomenology.

We focus in particular on the term in (6) that induces currents parallel to the magnetic field. Following Refs. [8,20] 
but keeping all terms, we find

$$
\begin{aligned}
\xi_{R, L}^{(B)}= & \frac{1}{2} C\left( \pm \mu_{R, L}-\frac{1}{2} \frac{n_{R, L}\left(\mu_{R}^{2}-\mu_{L}^{2}\right)}{\epsilon+P}\right) \\
& +\left( \pm T f^{\prime}\left(\frac{\mu_{R}-\mu_{L}}{T}\right)-\frac{n_{R, L} T^{2} f\left(\frac{\mu_{R}-\mu_{L}}{T}\right)}{\epsilon+P}\right)
\end{aligned}
$$

Here, the notation $\propto \pm \mu_{R, L}$ on the right-hand side is a shorthand denoting $+\mu_{R}\left(-\mu_{L}\right)$ for $\xi_{R}^{(B)}\left(\xi_{L}^{(B)}\right)$. In this compact notation, $f$ is an additional integration constant that, as first pointed out in [44], is not constrained by the thermodynamic consistency relations for $\xi_{R, L}^{(B)}$ [20]. The two terms proportional to $\frac{n_{R, L}}{\epsilon+P}$ arise from rewriting terms proportional to $\partial T / \partial \mu_{R, L}$ with the help of thermodynamic relations; we assume that these terms are much smaller than the other two terms in (7). More generally, we shall consider in the following situations with small charge densities and small chemical potentials, such that

$$
\begin{aligned}
v_{V}^{\mu} & =\left(\xi_{R}^{(B)}+\xi_{L}^{(B)}\right) B^{\mu}+O\left(\omega^{\mu}, \sigma\right) \simeq C \mu_{A} B^{\mu}, \\
v_{A}^{\mu} & =\left(\xi_{R}^{(B)}-\xi_{L}^{(B)}\right) B^{\mu}+O\left(\omega^{\mu}\right) \\
& \simeq\left(C \mu_{V}+2 T f^{\prime}\left(\frac{\mu_{A}}{T}\right)\right) B^{\mu} .
\end{aligned}
$$

Usually, discussions of experimental signatures neglect the unknown but thermodynamically allowed function $f\left(\frac{\mu_{A}}{T}\right)$ in Eqs. (7) and (8). Then, an external magnetic field induces an axial current in the presence of a finite vector charge density. This phenomenon is referred to as chiral separation effect (CSE). In addition, any time-dependent axial current will change the axial chemical potential $\mu_{A}$, and it will thus induce a vector current $v_{V}^{\mu}$. This is referred to as chiral magnetic effect (CME). The combination of both equations is the starting point for the discussion of the chiral magnetic wave (CMW).

One may wonder to what extent for small chemical potential and charge densities a nonvanishing function $f$ could change this picture. By construction, $v_{V}^{\mu}$ will remain unchanged, so charge separation along $B^{\mu}$ continues to take place for nonvanishing axial chemical potential. A negative term $\propto$ $T f^{\prime}\left(\frac{\mu_{A}}{T}\right)$, however, would counteract the induction of the axial current, and a positive contribution would enhance it. We note that for the vortical effect, a corresponding term was found to be nonvanishing in a holographic model [12]. Here, we follow common practice and we neglect contributions proportional to $f$, but we argue that a better understanding of these terms in QCD is desirable.

\section{A. A Bjorken-type model}

For the strongly expanding system created in heavyion collisions, the solution of the equations of motion of viscous relativistic fluid dynamics is complicated, and a fully realistic treatment requires numerical simulations. However, analytically treatable models, and in particular Bjorken-type models, have contributed in the past towards anticipating and understanding results of full fluid dynamical simulations. We expect that they can also play a useful role in understanding the dynamics of the QCD fluid coupled to strong external electromagnetic fields, where numerical studies are at the very beginning [33]. To this end, we formulate and study here a simplified fluid dynamical model of the chiral magnetic wave that retains essential aspects of a strongly expanding fluid.

In general, analytically treatable models of fluid dynamics assume additional symmetries in the initial conditions that are preserved during evolution and thus simplify the equations of motion. What is then simplified is not the dynamical treatment of the QCD fluid, but the description of the initial conditions under which this fluid is prepared. In practice, the symmetries assumed to this end in Bjorken-type models [45] are longitudinal boost invariance and invariance under azimuthal rotations or under transverse translations. If all these symmetries are invoked, one arrives at a 1+1-dimensional model that is analytically solvable in ideal and first-order viscous fluid dynamics. There is also a generalization of Bjorken-type models from Gubser [46], with analytical solutions of fluid dynamics that satisfy conformal symmetry.

Formulating a simplified model of the chiral magnetic wave is complicated because the phenomenon breaks all symmetries on which simplifications of fluid dynamics are typically based: A realistic magnetic field is not Bjorken boost invariant and the resulting fluid dynamic response breaks symmetry under rotations in the transverse plane. To arrive at a model requires one, therefore, to make simplifying assumptions not only about the initial conditions but also about the dynamical treatment of the QCD fluid. The main assumption in the following will be that the axial and vector charge densities and the corresponding chemical potentials are parametrically small so that one can treat them as symmetry-breaking perturbations that have a negligible effect on the energy density and pressure of the system. This assumption can be realized for a QCD fluid whose energy density is dominated by gluonic degrees of freedom, and for which net charge densities and associated chemical potentials are small. One can then consider energy density and pressure as providing parametrically large background fields for which initial conditions and subsequent evolution satisfy the symmetries of Bjorken's model to leading order in $\mu / T$. In particular, to leading order in $\mu / T$, the velocity $u^{\mu}$ that defines the rest frame of the energy density $\epsilon=u_{\mu} T^{\mu v} u_{v}$ takes at all space-time points the boost-invariant form,

$$
u^{\mu}=(t / \tau, 0,0, z / \tau)=(\cosh \eta, 0,0, \sinh \eta),
$$

where $z$ is the beam direction, $\tau$ the proper time, and $\eta$ the space-time rapidity. Charges propagate as small perturbations (subleading in $\mu / T$ ) on top of this Bjorken background field, and their propagation is seen in the dissipative parts $v_{R, L}^{\mu}$ of the currents as well as in the position dependence of the charge densities $n_{R, L}$ in (4). It is only these latter terms that break the symmetries of the Bjorken model in response to an external magnetic field.

For a constant external magnetic field $B_{x}$, we can write to leading order in $\mu / T$ Eqs. (1)-(6) as four equations for the energy density $\epsilon$ and pressure $P$ (we use $\bar{\mu}_{R, L} \equiv \mu_{R, L} / T$ ),

$$
\begin{array}{r}
\partial_{\tau} \epsilon+\frac{\epsilon+P}{\tau}-\frac{\frac{4}{3} \eta_{s}+\zeta}{\tau^{2}} \\
=B_{x} \sigma T \sinh \eta \partial_{y} \bar{\mu}_{V},
\end{array}
$$

$\partial_{x} P=0$ 


$$
\begin{aligned}
& \partial_{y} P=B_{x} \sigma T \frac{1}{\tau}\left(\cosh \eta \partial_{\eta} \bar{\mu}_{V}-n_{V} \sinh \eta\right), \\
& \partial_{\eta} P=-B_{x} \sigma T \tau \partial_{y} \bar{\mu}_{V},
\end{aligned}
$$

and two equations for the time evolution of the charge densities,

$$
\partial_{\tau} n_{R, L}+\frac{n_{R, L}}{\tau}-\sigma \vec{\nabla}\left(T \vec{\nabla} \bar{\mu}_{R, L}\right)+B_{x} \cosh \eta \partial_{x} \xi_{R, L}^{(B)}=0 .
$$

These equations are not yet self-consistent because they were derived assuming longitudinal boost invariance for $u^{\mu}, P$, and $\epsilon$ while they contain terms with explicit $\eta$ dependence that break longitudinal boost invariance. The reason for this is, of course, that (10)-(14) were derived for a constant magnetic field $B_{x}$ that breaks longitudinal boost invariance. To obtain a consistent, longitudinally boost-invariant set of differential equations, we could choose either a magnetic field of longitudinally boost-invariant form that coincides at $\eta=0$ with the physical value of $B_{x}$ at midrapidity. This would give rise to a consistent dynamics that is physically meaningful at $\eta=0$. Equivalently, we can simply limit our discussion of Eqs. (10)-(14) to the transverse plane at spacetime rapidity $\eta=0$, for which all terms on the right-hand side of Eqs. (10)-(13) vanish. For the terms $\propto \partial_{y} \bar{\mu}_{V}$ and $\propto \partial_{\eta} \bar{\mu}_{V}$, this follows from the fact that even in the presence of a constant magnetic field, the system is symmetric under translation in the transverse direction orthogonal to the magnetic field and under reflection $\eta \rightarrow-\eta$. For $\eta=0$, we, therefore, find for energy density and pressure the equations of motion of the Bjorken model to first order in viscous hydrodynamics,

$$
\begin{aligned}
\partial_{\tau} \epsilon+\frac{\epsilon+P}{\tau}-\frac{\frac{4}{3} \eta_{s}+\zeta}{\tau^{2}} & =0, \\
\vec{\partial} P & =0,
\end{aligned}
$$

supplemented by a simple time evolution for the charge densities,

$$
\partial_{\tau} n_{R, L}+\frac{n_{R, L}}{\tau}-\sigma \nabla^{2} \mu_{R, L} \pm B_{x} \partial_{x} \xi_{R, L}^{(B)}=0 .
$$

Equations (15)-(17) are the starting point of the following discussion. We recall that they provide a simplified consistent dynamical treatment if restricted to midrapidity. The following analytical and numerical studies will be limited to the case where the energy density is translationally invariant in the transverse $(x, y)$ plane and where the charge distributions are invariant in the transverse direction $y$ orthogonal to the direction of the magnetic field. The advantage of translation invariance with respect to $y$ is that we arrive at a very transparent model for the efficiency of electric charge separation via the CMW.

\section{B. The chiral magnetic wave in an expanding fluid}

Equation (17) describes the hydrodynamic propagation of chiral charge density in a Bjorken-expanding background. To make contact with earlier discussions of the chiral magnetic wave, we work in the limit of small chemical potential when

$$
\mu_{R, L} \simeq 2 \alpha n_{R, L},\left.\quad \alpha \equiv \frac{\partial \mu_{R, L}}{2 \partial n_{R, L}}\right|_{n_{R, L}=0} .
$$

Keeping only the dominant first term of Eq. (7), $\xi_{R, L}^{(B)}=$ $\pm \frac{1}{2} C \mu_{R, L}$, we obtain

$$
\partial_{\mu}\left(n_{R, L} u^{\mu}\right) \pm B_{x} C \alpha \partial_{x} n_{R, L}-2 \sigma \alpha \Delta n_{R, L}=0 .
$$

According to this equation of motion, right-handed (lefthanded) charge density is transported with velocity,

$$
v_{x}=B_{x} C \alpha,
$$

along (opposite) the magnetic field. This charge transport is accompanied by diffusion characterized by the diffusion constant,

$$
D=2 \sigma \alpha
$$

We note that Eq. (19) derived here is a natural generalization of the equation for a chiral magnetic wave written in Refs. [30,31] for the case of a static medium. Indeed, inserting the velocity profile for a time-independent medium, $u^{\mu}=(1,0,0,0)$, into (19) one finds the form discussed in Refs. [30,31],

$$
\left(\partial_{t} \pm v_{x} \partial_{x}-D \partial_{x}^{2}\right) n_{R, L}=0 . \quad \text { (static medium) }
$$

The main features of charge transport in a static medium can be inferred from general considerations without explicit calculation. In particular, one knows that the velocities $u^{\mu}, u_{(\mathrm{E})}^{\mu}$ of the fluid dynamic frames comoving with energy density (Landau frame) and with a charge density (Eckhart frame) are related in first-order hydrodynamics by the linear relation (see, e.g., Ref. [47]),

$$
u_{R, L(\mathrm{E})}^{\mu}=u^{\mu}+\frac{v_{R, L}^{\mu}}{n_{R, L}} .
$$

The velocities of charge transport are then given by the difference between the velocity that defines the Landau frame of comoving energy density and the Eckhart frames of comoving left(+)- or right(-)-handed charge densities. Inserting the above relations, one checks easily the expectation that the velocity of charge transport,

$$
\pm v_{x}=u_{R, L(\mathrm{E})}^{\mu}-u^{\mu}=\frac{1}{2} C \frac{\mu_{R, L}}{n_{R, L}} B_{x}=B_{x} C \alpha,
$$

is determined by the transverse boost from the Landau frame of comoving energy density to the Eckhart frame that comoves with left $(+)$ - or right $(-)$-handed charges. The velocity $v_{x}$ of charge transport derived for the Bjorken-type model in (20) equals that obtained from this general consideration. In Refs. [30,31], also the presence of a diffusion term $\propto \Delta n_{R, L}$ was argued for on physical grounds. In summary, we have shown in this subsection that the fluid dynamic equations of motion (15)-(17) are a natural generalization of the chiral magnetic wave equation of Refs. [30,31] to the case of a Bjorken-expanding background field, and we have recalled how the physics encoded in these equations is related to general considerations about charge transport. 


\section{SOLUTIONS TO THE CMW IN AN EXPANDING BACKGROUND}

In general, solutions to the fluid dynamic equations of motion depend on the initial conditions for all fluid dynamic fields, the equation of state and the value of transport coefficients, and the strength of external fields. As for the equation of state, there are lattice simulations [48] that determine for two-flavor QCD up to sixth $\operatorname{order}(k=3)$ the coefficients $C_{k}$ in the power series,

$$
P\left(T, \mu_{R}, \mu_{L}\right)=T^{4} \sum_{k} C_{k}\left[\left(\frac{\mu_{R}}{T}\right)^{2 k}+\left(\frac{\mu_{L}}{T}\right)^{2 k}\right] .
$$

As we shall work in the present case for small chemical potentials only, we shall focus on the first two terms in this series, setting $C_{k}=0$ for $k \geqslant 2$. This is also justified by the observation in Ref. [48] that the coefficients $C_{k}$ of higher orders $k \geqslant 1$ are numerically smaller by factors $O\left(10^{-k}\right)$. Numerically, it was found that at temperatures well above $T_{c}$, the coefficients $C_{0}$ and $C_{1}$ are approximately $80 \%$ of the value of a free gas of gluons and $n_{f}=2$ quarks [48]. This is in line with results for field theories with gravity duals for which the equation of state is known to vary between the limits of vanishing and infinite coupling by a constant factor $3 / 4$ [49] or by a factor close to $3 / 4$ [50]. For numerical studies, we shall therefore choose (25) with

$$
\begin{aligned}
C_{0} & =0.8\left(\frac{8 \pi^{2}}{45}+\frac{\pi^{2}}{15} \frac{7}{4} n_{f}\right), \\
C_{1} & =0.8 \frac{1}{4} n_{f} .
\end{aligned}
$$

Furthermore, to allow for analytical calculations, we consider in the following an ideal equation of state $\epsilon=3 P$. For the purpose of a numerical study of the chiral magnetic wave, an ideal equation of state with pressure (25)-(27) is a transparent but simplified starting point. We note in particular that the equation of state will depend in general also on the external magnetic field. Recent studies indicate significant changes of the equation of state in a magnetic field. For instance, the chiral and deconfinement phase transitions may split [51,52] and one finds a magnetization of the QCD plasma [53]. However, in these works and in the references cited therein, the natural scale for the onset of significant modifications of the equation of state from B fields is $e B>0.1 \mathrm{GeV}^{2} \approx 10 m_{\pi}^{2}$. As we shall see in the following discussion, the external magnetic field strength realized in ultrarelativistic heavy-ion collisions lies below this scale. Because we study in the following scenarios with $e B<10\left(m_{\pi}\right)^{2}$ only, we shall neglect possible effects of the Landau quantization of the energy spectrum of quarks on the equation of state at finite $B$, and we shall work with the ansatz (25)-(27).

With the help of the thermodynamic relation, $d P=s d T+$ $n_{R} d \mu_{R}+n_{L} d \mu_{L}$ and the equation of state (25), we can write the equations of motion (15)-(17) as coupled partial differential equations for the temperature and the chemical potentials $\mu_{R}$ and $\mu_{L}$. Using $\mathcal{M}^{2} \equiv \mu_{R}^{2}+\mu_{L}^{2}$, we write

$$
\begin{aligned}
& \left(2 C_{0} T^{3}+C_{1} T \mathcal{M}^{2}\right) \partial_{\tau} T+\frac{1}{2} C_{1} T^{2} \partial_{\tau} \mathcal{M}^{2} \\
& +\frac{2}{3 \tau}\left(C_{0} T^{4}+C_{1} T^{2} \mathcal{M}^{2}\right)=\frac{4 \frac{\eta}{s}}{9 \tau^{2}}\left(2 C_{0} T^{3}+C_{1} T \mathcal{M}^{2}\right),
\end{aligned}
$$

and

$$
\begin{gathered}
\partial_{\tau} \mu_{R, L}+\frac{\mu_{R, L}}{\tau}+2 \frac{\mu_{R, L}}{T} \partial_{\tau} T \pm \frac{1}{4} \frac{C}{C_{1}} \frac{B_{x}}{T^{2}} \partial_{x} \mu_{R, L} \\
-\frac{\sigma}{2 C_{1} T^{2}} \partial_{x}^{2} \mu_{R, L}=0 .
\end{gathered}
$$

These equations of motion can be solved numerically for arbitrary initial conditions, and we shall discuss such numerical results in the following. First, however, we turn to an approximate analytical solution that will turn out to illustrate the main features of the full numerical result.

\section{A. Approximate analytical solution for Gaussian initial condition}

In the limit of small charge densities and negligible viscosity, the solution to the equation of motion (28) is the Bjorken solution up to parametrically small corrections,

$$
T=T_{0}\left(\frac{\tau_{0}}{\tau}\right)^{1 / 3}+O\left(\frac{\mu_{R, L}}{T}, \frac{\eta}{S}\right) .
$$

Entering with this expression Eq. (29) and neglecting all nonlinear, higher order terms in the charge density, one finds

$$
\partial_{\tau} \tilde{\mu}_{R, L} \pm \frac{C}{4 C_{1}} \frac{\tau^{2 / 3} B_{x}}{T_{0}^{2} \tau_{0}^{2 / 3}} \partial_{x} \tilde{\mu}_{R, L}-\frac{\tau^{2 / 3} \sigma}{2 C_{1} T_{0}^{2} \tau_{0}^{2 / 3}} \partial_{x}^{2} \tilde{\mu}_{R, L}=0 .
$$

Here, we have separated the leading time dependence of the charge densities, that is from longitudinal Bjorken expansion,

$$
\mu_{R, L}(\tau, x)=\tilde{\mu}_{R, L}(\tau, x)\left(\frac{\tau_{0}}{\tau}\right)^{1 / 3} .
$$

We consider first initial conditions of heavy-ion collisions that show event-by-event an initially negligible density $n_{A}$ of axial charges but a nonvanishing density of electric charges of approximately Gaussian shape, centered at initial time $\tau_{0}$ at transverse position $x=0$. We want to understand how this electric charge distribution evolves from the effects of the QCD anomaly in the presence of a large and time-dependent magnetic field $B_{x}(\tau)$. The fluid dynamic problem studied here is simpler than the situation in heavy-ion collisions in that it ignores transverse gradients in energy density and pressure and thus retains a one-dimensional translational symmetry in the transverse direction $y$. However, the problem is sufficiently complex to study the effects of charge separation along the magnetic field in a Bjorken expanding fluid. Interestingly, while the discussion of (31) for general initial conditions requires numerical techniques, the solution for a Gaussian initial density distribution can be given analytically,

$$
\mu_{R, L}(\tau, x)=\left(\frac{\tau_{0}}{\tau}\right)^{1 / 3} \frac{\text { const. }}{R(\tau)} \exp \left[-\frac{1}{2} \frac{\left(x+s_{ \pm}(\tau)\right)^{2}}{R^{2}(\tau)}\right] .
$$


Here, the peak of the Gaussian distribution for right- and lefthanded charges evolves in time according to

$$
s_{ \pm}(\tau)=\mp \frac{1}{4} \frac{C}{C_{1}} \frac{\tau_{0}^{-2 / 3}}{T_{0}^{2}} \int_{\tau_{0}}^{\tau} \tau^{2 / 3} B_{x}\left(\tau^{\prime}\right) d \tau^{\prime} .
$$

For the equation of state (25), one finds $n_{R, L}=2 C_{1} T^{2} \mu_{R, L}$ and therefore $\alpha=\frac{\partial \mu_{R, L}}{2 \partial n_{R, L}}=1 / 4 C_{1} T^{2}$. From this, one checks easily that the position of the peak moves with the expected velocity (20),

$$
\frac{d s_{ \pm}(\tau)}{d \tau}=v_{x}(\tau)=B_{x}(\tau) C \alpha .
$$

The spatial separation $s_{+}-s_{-}$of right- and left-handed charges is of direct phenomenological relevance. In the fluid dynamic model studied here, this separation is proportional to a particular time average of the time-dependent external magnetic field, $\int_{\tau_{0}}^{\tau} \tau^{\prime 2 / 3} B_{x}\left(\tau^{\prime}\right) d \tau^{\prime}$. One may wonder whether this finding is valid also outside the model-dependent framework in which we have derived it here. In this context, we note that for small charge densities, one generally expects a linear relation between chemical potentials and charges. In the absence of other dimensionful parameters, this dependence must be of the form $n_{R, L} \propto T^{2} \mu_{R, L}$, and therefore $\alpha \propto 1 / T^{2}$. For an expanding fluid, it is then the time dependence of the dimensionless ratio $B_{x}(\tau) / T^{2}(\tau)$ that determines the time dependence of the velocity $v_{x}(\tau)$ with which right- and left-handed charges separate. The time dependence of the integrand of (34) is therefore generally expected for fluids with Bjorken expansion. We note as an aside that for the case of a fluid with transverse expansion, when one expects $T(\tau)=T_{0}\left(\tau_{0} / \tau\right)^{\delta}, 1 / 3<\delta<1$, these considerations suggest that the spatial separation $s_{+}-s_{-}$will depend on a weighted time average $\int_{\tau_{0}}^{\tau} \tau^{2 \delta} B_{x}\left(\tau^{\prime}\right) d \tau^{\prime}$ of the magnetic field.

The square of the Gaussian width in (33) broadens according to the differential equation $\partial_{\tau}\left(\frac{1}{R^{2}}\right)=-\frac{\sigma}{C_{1} T^{2}}\left(\frac{1}{R^{2}}\right)^{2}$. In the present paper, we consider mainly the case of a temperatureindependent electrical conductivity, for which one finds

$$
\left.R^{2}(\tau)\right|_{\sigma=\text { const. }}=R^{2}\left(\tau_{0}\right)+\frac{3}{5} \frac{\sigma}{C_{1} T_{0}^{2} \tau_{0}^{2 / 3}}\left(\tau^{5 / 3}-\tau_{0}^{5 / 3}\right) .
$$

We note, however, that in calculations of the conductivity $\sigma$ of conserved $U(1)$ charges in theories with gravity dual, one finds a linear dependence on temperature, $\sigma=\sigma_{0} T[54,55]$, and this is also expected on dimensional grounds. Including in the calculation of $R^{2}(\tau)$ the time dependence of $\sigma$ that would result from a linear temperature dependence, one finds instead of (36) a slightly weaker time dependence,

$$
\left.R^{2}(\tau)\right|_{\sigma=\sigma_{0} T}=R^{2}\left(\tau_{0}\right)+\frac{3}{4} \frac{\sigma_{0}}{C_{1} T_{0} \tau_{0}^{1 / 3}}\left(\tau^{4 / 3}-\tau_{0}^{4 / 3}\right) .
$$

This illustrates that details of the diffusion will depend on the temperature dependence of the conductivity. However, these effects turn out to be numerically small, and we therefore fix in the following the conductivity to a value $\sigma=$ $e^{2} 200 \mathrm{MeV}$ that is consistent with the recent (quenched) lattice QCD studies [56], $\frac{1}{3} e^{2} T_{c}<\sigma<e^{2} T_{c}$, where $e^{2}=4 \pi / 137$.

\section{B. Numerical results for initial Gaussian vector charge distribution}

We start by summarizing the input used in the following numerical study of the fluid dynamic equations of motion (15)-(17) of the chiral magnetic wave. We use the ideal equation of state $\epsilon=3 P$ with pressure given in Eq. (25) and with nonvanishing coefficients (26) and (27). The default values for the ratio of shear viscosity over entropy and for the electrical conductivity are $\eta / s=1 / 4 \pi$ [57] and $\sigma=$ $e^{2} 200 \mathrm{MeV}$, respectively. As default, we initialize the system with a temperature $T\left(\tau_{0}=0.1 \mathrm{fm} / \mathrm{c}\right)=T_{0}=650 \mathrm{MeV}$ that corresponds for a fluid with Bjorken expansion to the initial temperature $T\left(\tau_{0}=0.6 \mathrm{fm} / \mathrm{c}\right)=360 \mathrm{MeV}$ consistent with fluid dynamic simulations of heavy-ion collisions at RHIC en$\operatorname{ergy}\left(\sqrt{s_{\mathrm{NN}}}=200 \mathrm{GeV}\right)$. We initialize the chemical potentials with a Gaussian profile (33) of transverse width $R=3 \mathrm{fm}$. We use an initial strength $\mu_{R, L}\left(\tau_{0}, x=0\right)=10 \mathrm{MeV}$ consistent with the assumption that the contribution of charges to the pressure of the system is perturbatively small, $\mu / T \ll 1$. We note, however, that the dependence on $\mu_{R, L}$ is linear for sufficiently small charge densities, and most of our results will be normalized such that they are independent of $\mu_{R, L}\left(\tau_{0}, x=\right.$ $0)$. For the strength of the abelian anomaly, we use $C=\frac{e}{2 \pi^{2}}$; this means that we do not take the fractional charge of quarks into account.

One aim of the following study is to arrive at some qualitative statement about how phenomenologically testable effects of the chiral magnetic wave depend on the center-ofmass energy. To allow for this discussion, we include in the calculation the $\sqrt{s_{\mathrm{NN}}}$ dependence of the initial temperature by requiring that the entropy of the system matches the $\sqrt{s_{\mathrm{NN}}}$ dependence of the measured charged particle multiplicity at midrapidity, $T^{3}(\tau=$ fixed $)=$ const. $d N_{\mathrm{ch}} / d \eta$. For the event multiplicities in heavy-ion collisions published in Refs. [58,59], this implies that in comparison to the upper RHIC energy $\left(\mathrm{Au}+\mathrm{Au}\right.$ at $\left.\sqrt{s_{\mathrm{NN}}}=200 \mathrm{GeV}\right)$, the initial temperature $T_{0}$ at fixed $\tau_{0}$ is a factor $\sim 1.35$ larger at LHC $\left(\mathrm{Pb}+\mathrm{Pb}\right.$ at $\left.\sqrt{s_{\mathrm{NN}}}=2.76 \mathrm{TeV}\right)$ and a factor $\sim 0.81$ smaller at lower RHIC or upper SPS energy of $\sqrt{s_{\mathrm{NN}}}=17 \mathrm{GeV}$. Moreover, we choose for the external magnetic field in our calculations the dependence on center-of-mass energy $\sqrt{s_{\mathrm{NN}}}$, proper time $\tau$, and impact parameter $b$ expected for realistic heavy-ion collisions. We calculate the strength of this magnetic field from the distributions of electric charges of spectators and participants according to the classical electrodynamic calculation described in the appendix of Ref. [22]; see Fig. 1.

The $\sqrt{s_{\mathrm{NN}}}$ dependence of the maximal value of the magnetic field strength and the temporal fall-off shown in Fig. 1 is in rough quantitative agreement with numerical results of a microscopic transport model [60] for fixed target and RHIC energies, and with calculations for RHIC and LHC energies [61]. In comparison to a model of hadron string dynamics [62], Fig. 1 overestimates the magnetic field strength somewhat. On the other hand, it was demonstrated in model studies that event-wise fluctuations in the incoming charge distributions can lead to larger values of the magnetic field strength in some events $[43,63]$, and that they can also lead to very large fluctuations in the electric field strength. 


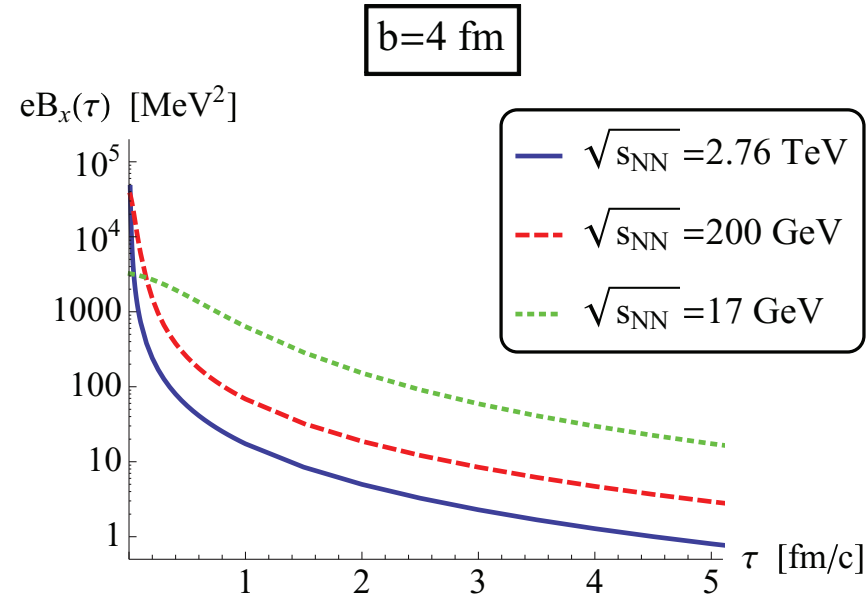

(a)

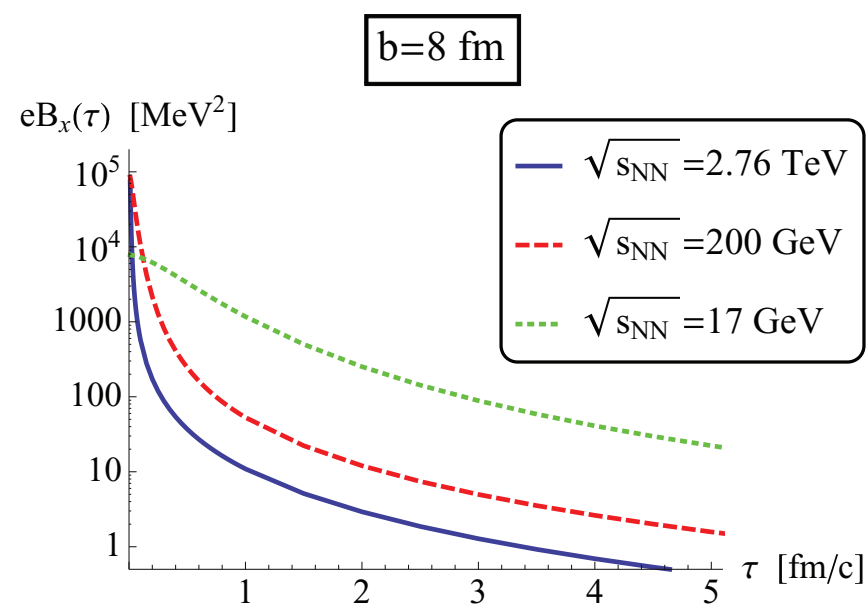

(b)

FIG. 1. (Color online) Time-dependent strength of the transverse component of the external magnetic field obtained in a classical electromagnetic calculation [22] field for nucleus-nucleus collisions at different center-of-mass energies and for different impact parameter $b$.

In summary, these findings suggest that the time-dependent magnetic field strengths plotted in Fig. 1 provide reasonable ballpark estimates for the following discussion. One may wonder whether this conclusion could change significantly because of the effects of magnetization of the QGP that may prolong the lifetime of the magnetic field [64]. For this to happen, one would have to require a dynamical mechanism able to trap magnetic fields of strength $>10^{17}$ Gauss in a fluid of quarks and gluons. A recent analysis suggests that at least for the values of the electric conductivity obtained from lattice QCD calculations, such effects of magnetization are relatively small [65]. In the following numerical studies, we therefore use the strength and time dependence of the $B_{x}$ field in Fig. 1. Because the dependence on impact parameter is relatively mild, we show results only for a default value of the $B_{x}$ field at $b=4 \mathrm{fm}$. In the present model, this magnetic field is position independent and permeates the fluid at midrapidity.

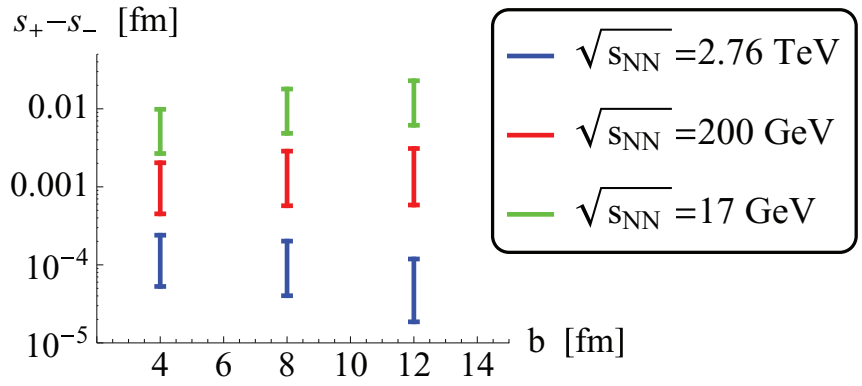

FIG. 2. (Color online) Spatial separation of right- and lefthanded axial charge, occurring according to Eq. (34) in the magnetic field of Fig. 1 between times $\tau_{0}$ and $\tau=10 \mathrm{fm} / \mathrm{c}$. The upper (lower) end of the uncertainty band corresponds to initial value $\tau_{0}=0.01$ $\mathrm{fm} / \mathrm{c}(0.1 \mathrm{fm} / \mathrm{c})$.

Figure 1 shows clearly that while the peak strength of the magnetic field increases with increasing center-of-mass energy, its temporal fall-off is much faster for larger $\sqrt{s_{\mathrm{NN}}}$. As a consequence, the external magnetic field at upper CERN SPS and lower RHIC energy of $\sqrt{s_{\mathrm{NN}}}=17 \mathrm{GeV}$ exceeds from times $\tau>0.1 \mathrm{fm} / \mathrm{c}$ onwards the corresponding external fields attained at upper RHIC and LHC energies. Whether phenomenologically testable effects of the chiral magnetic wave are maximized at higher or lower center-of-mass energy thus becomes a dynamical question. To inform us about this point, we plot in Fig. 2 the spatial separation $s_{+}-s_{-}$ of right- and left-handed charges, evaluated according to Eq. (34) for different centralities and different center-of-mass energies. Before commenting on the absolute size of the spatial separation, let us comment on the observed dependencies. In particular, we find that the spatial separation is larger at smaller center-of-mass energy. We recall that the velocity of charge separation in (34) is $v_{x}(\tau) \propto B_{x}(\tau) / T^{2}(\tau)$. This explains that the $\sqrt{s_{\mathrm{NN}}}$ dependence of $s_{+}-s_{-}$seen in Fig. 2 is mainly from the weaker temporal fall-off of the magnetic field at small $\sqrt{s_{\mathrm{NN}}}$, but the fact that the temperature at fixed time $\tau$ decreases with decreasing $\sqrt{s_{\mathrm{NN}}}$ does also play a role. Because $B_{x}(\tau)$ peaks at very small times, there is a significant $\tau_{0}$ dependence of $s_{+}-s_{-}$. Initializing a hydrodynamic calculation at too short times $\tau_{0}$ is certainly questionable. However, to quantify the uncertainty in varying $\tau_{0}$, it is useful to note that the integral $\int_{\tau_{0}}^{\tau} \tau^{\prime 2 / 3} B_{x}\left(\tau^{\prime}\right) d \tau^{\prime}$ in (34) stays finite even in the limit $\tau_{0} \rightarrow 0$. For this reason, we plot in Fig. 2 upper values that correspond to maximizing the contribution of this integral by choosing a very small lower integration boundary $\tau_{0}=0.01 \mathrm{fm} / \mathrm{c}$. Even with this optimistic ansatz, the spatial separation of rightand left-handed charges during the evolution is only of order $O\left(10^{-2} \mathrm{fm}\right)$ at SPS energies and it is one (two) orders smaller at RHIC (LHC) energies. This raises the question under which circumstances phenomenological consequences of the chiral magnetic wave may become testable.

We now turn to the discussion of numerical solutions of the equations of motion (28) and (29) for the CMW. We first consider an initial condition that corresponds along the direction $x$ of the magnetic field to a Gaussian distribution of vector charge $n_{V}=n_{R}+n_{L}$ and a vanishing distribution 


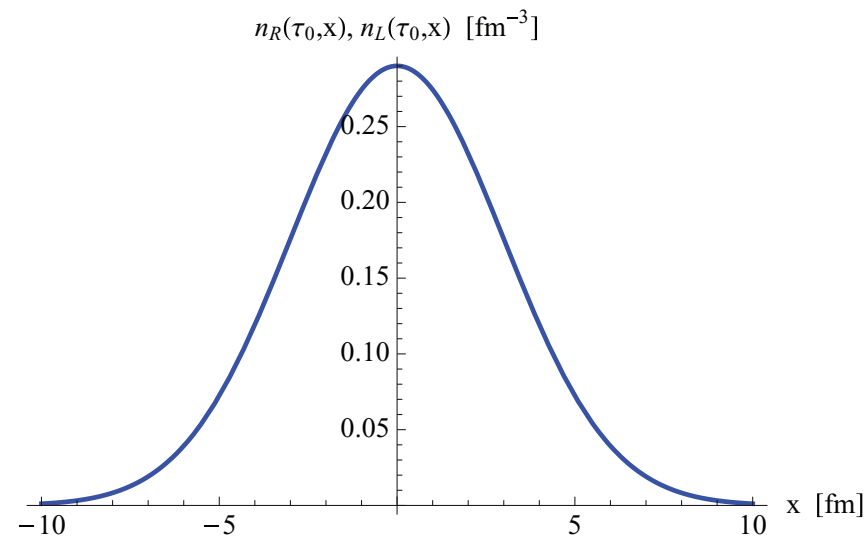

FIG. 3. (Color online) Initial condition for the distribution of charge densities $n_{R}, n_{L}$, corresponding to Gaussian distribution (33) with norm $\mu_{R, L}\left(\tau_{0}, x=0\right)=10 \mathrm{MeV}$ and Gaussian width $R\left(\tau_{0}\right)=$ $3 \mathrm{fm}$.

of axial charge $n_{A}=n_{R}-n_{L}$. Figure 3 shows this distribution for the default values of the thermodynamic variables discussed above. One sees that the default values chosen here correspond to a small charge density, consistent with the idea that we can treat charge transport as a perturbation on top of a dynamically evolving background. According to Fig. 2 and the analytical considerations of Sec. III A, we expect that the peak of the Gaussian distribution evolves very little with time. For clarity, we therefore follow the procedure of Ref. [30] and we subtract from the time-evolved vector charge distribution $n_{V}$ the vector charge distribution without chiral magnetic wave, that is, the distribution $\left.n_{V}\right|_{B=0}$ obtained from evolving Fig. 2 in the absence of a magnetic field. In Fig. 4, we show this difference normalized to the time-dependent vector charge density at the center $x=0$. The top panel of Fig. 4 shows how the chiral magnetic wave separates vector charge density in the transverse plane with increasing time. The middle and lower panel of Fig. 4 shows that our analytical solution (33)-(35), although being accurate only to leading order in $\mu / T$, accounts for the full numerical result of the normalized charge difference $\left(n_{V}-\left.n_{V}\right|_{B=0}\right) /\left.n_{V}\right|_{B=0}$ very well. To this end, we show first that analytical and numerical results for this ratio coincide almost for vanishing shear viscosity, and that a finite value of $\eta / s$ reduces the effect only mildly. We then show in the lower panel that the analytical result is highly sensitive to the value of the transverse velocities $v_{ \pm}$that determine the norm of the spatial separation $s_{ \pm}$in (34). This demonstrates that Eq. (34) and the spatial charge separation plotted in Fig. 2 provides a quantitative basis for understanding the size of the effect. Figure 5 then shows how the corresponding asymmetry of the axial charge distribution $n_{A}$ develops with time. Because this distribution vanishes at $\tau_{0}$ for the initial condition of Fig. 3 studied here, we normalize the distribution to $\left.n_{V}\right|_{B=0}$.

Distributions for $n_{V}-\left.n_{V}\right|_{B=0}$ and $n_{A}$ at times $\tau=10 \mathrm{fm}$ were shown previously in Figs. 1 and 2 of Ref. [30]. In contrast to the present study, these results were obtained for a static medium and a constant time-independent magnetic field of strength $e B=m_{\pi}^{2}$ which corresponds approximately to the peak value of $e B_{x}(\tau)$ in Fig. 1. In addition, this study

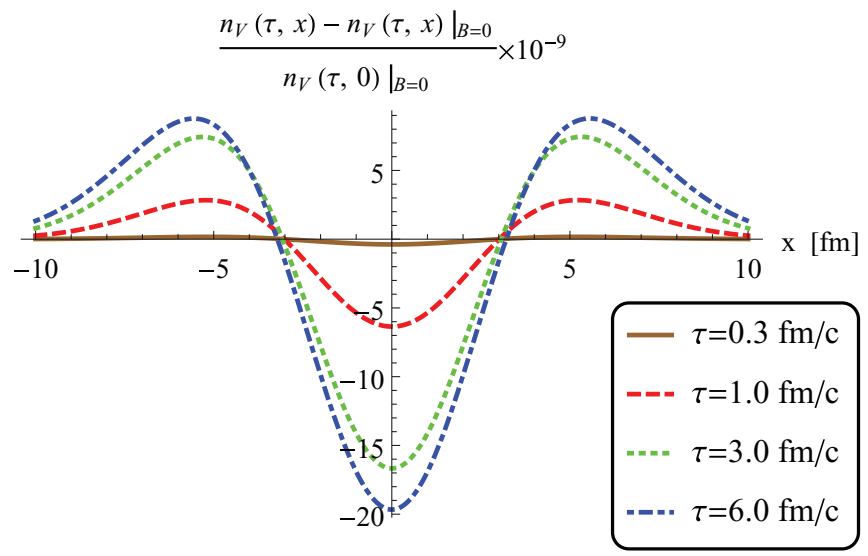

(a)

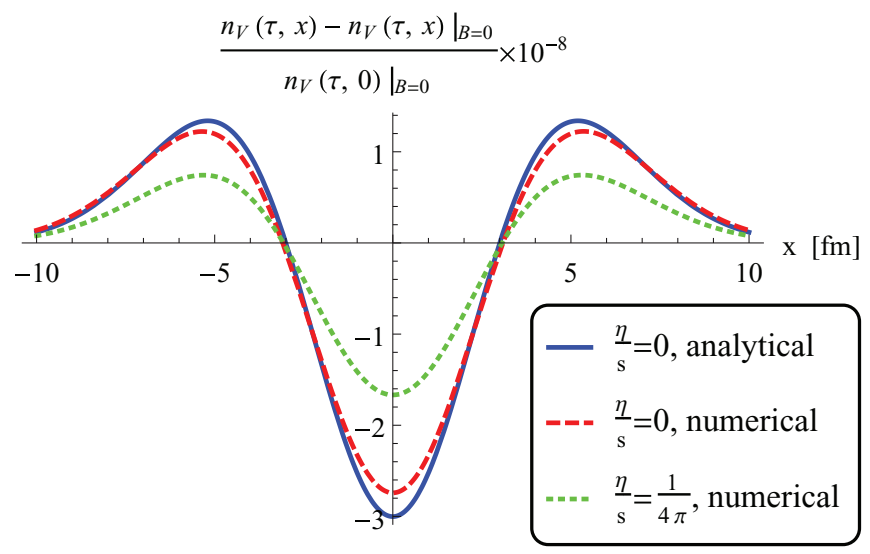

(b)

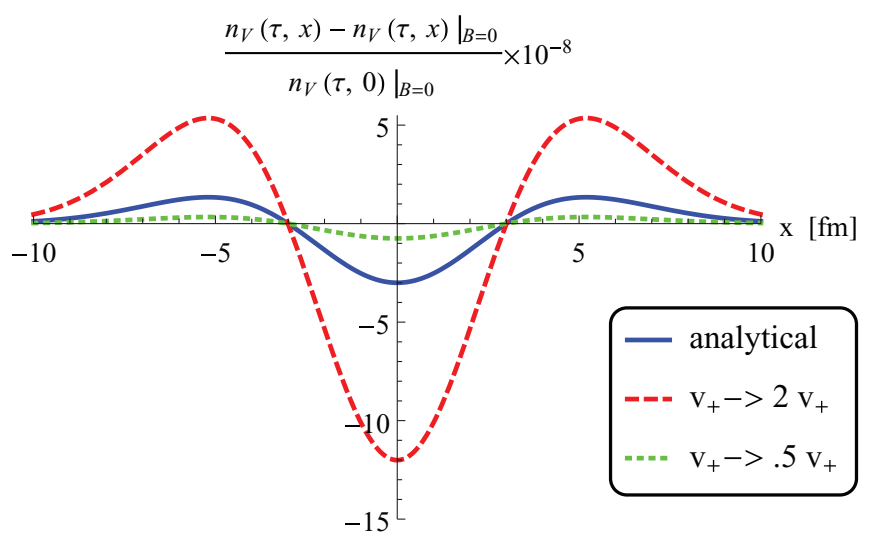

(c)

FIG. 4. (Color online) The normalized and subtracted vector charge distribution for initial conditions corresponding to $b=4 \mathrm{fm}$ at $\sqrt{s_{\mathrm{NN}}}=17 \mathrm{GeV}$ and initial charge distributions shown in Fig. 3. (a) Numerical solution of (28) and (29) for different times into the evolution. (b) Comparison of the analytical result (34)-(36) with the full numerical solution at vanishing and at finite shear viscosity. (c) Dependence of the analytical result on the analytically determined transverse velocity of charge transport.

in Ref. [30] was for a smaller constant temperature $T=$ $165 \mathrm{MeV}$ which according to our discussion helps to maximize the effect. Reference [30] set the initial axial chemical potential 


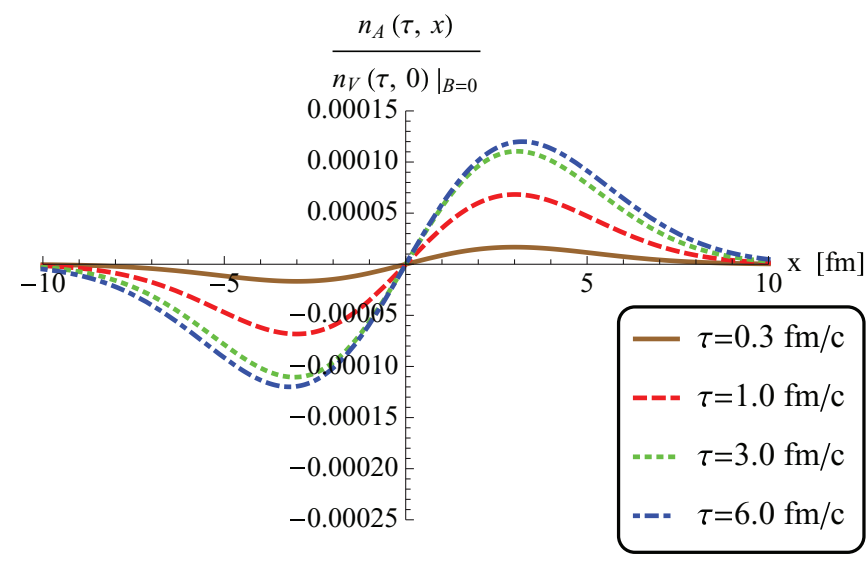

FIG. 5. (Color online) The normalized axial charge distribution determined for the same input as in Fig. 4(a).

to zero, consistent with the initial conditions considered here. They initialized a two-dimensional vector chemical potential with a Woods-Saxon profile and they evolved the chiral magnetic wave Eq. (22) numerically. Despite these qualitative and quantitative differences, we observe that if one cuts the resulting two-dimensional distributions $n_{V}-\left.n_{V}\right|_{B=0}$ and $n_{A}$ of Ref. [30] at transverse position $y=0$ along the direction of the magnetic field, their shape and the transverse positions of their peaks resembles closely the results shown in Figs. 4 and 5 of the present work.

Given that the transverse displacement $s_{+}-s_{-}$of charges is very small (see Fig. 2), it is clear that the positions of the peaks of the distributions in Figs. 4 and 5 cannot be of dynamical origin alone. Rather, irrespective of the exact size of $v_{ \pm}$and of the time dependence of $s_{+}-s_{-}$that is sensitive to the chiral magnetic wave, the peaks in the distribution of Figs. 4 and 5 will always be positioned at approximately twice the Gaussian width of $n_{V}\left(\tau_{0}\right)$, because they result from subtracting two equally normalized Gaussian distributions with almost the same width. This conclusion may be expected to depend only mildly on whether the shape of the charge distribution is Gaussian or Woods-Saxon, and this may explain the similarity between our results and those of Ref. [30]. As for the size of the effect, we note that the results of Figs. 4 and 5 are normalized such that they are independent of the absolute value of the charge density. According to our results, the effective charge transport caused by the chiral magnetic wave affects only a very small fraction $O\left(10^{-9}-10^{-10}\right)$ of all the vector charges at midrapidity. Similarly, Fig. 5 indicates that the dynamical buildup of an axial charge asymmetry is a numerically very small effect, at least for the input parameters considered here. One may wonder whether one can obtain numerically larger effects by changing input parameters. Given that the size of the effect is clearly governed by the integral (34), the only way of maximizing the effect is to go to even larger magnetic fields and/or to even smaller temperatures than those estimated for collisions at SPS energy. The results found for upper RHIC (LHC) energy are similar in shape to those in Figs. 4 and 5, but they are approximately one (two) orders of magnitude smaller in size (data not shown). Here, we note that even if we choose a time-independent magnetic $e B=m_{\pi}^{2}$, the distribution in Fig. 4 does not rise larger than peak values of $O\left(10^{-5}\right)$ which is still less than one charged particle per collision.

\section{INITIAL CONDITIONS WITH FINITE AXIAL CHEMICAL POTENTIAL}

For a fluid dynamic evolution initialized with vanishing axial charge density, we have seen in Sec. III that the chiral magnetic wave is unlikely to generate an experimentally testable electric quadrupole moment within the mesoscopic and finite-lived systems created in ultrarelativistic heavy-ion collisions. In our studies, the fraction of the total charge density, separated by the CMW along the direction of $B_{x}$ turned out to be several orders of magnitudes too small, even under optimistic choices of thermodynamic variables; see Fig. 4. We have traced back this finding to the parametrically and numerically small value of the transverse velocity $v_{x}$ of charge transport, which leads to spatial separations of vector charge that are typically much smaller than $1 \mathrm{fm}$; see Fig. 2.

We now investigate to what extent this conclusion can change for initial conditions with nonvanishing axial charge asymmetry in the direction of $B_{x}$. The physical motivation for exploring this possibility is twofold. First, for a nonvanishing initial axial charge density distribution as the one shown in the lower panel of Fig. 6, vector charge separation can be expected to be dynamically more efficient because a sizable finite axial chemical potential needed for vector charge transport is present from $\tau_{0}$ onwards and does not need to be generated dynamically from gradients of the vector charge distribution. Second, as pointed out in particular in the recent works of Refs. [32,33], the nonconservation of the axial current $\partial_{\mu} j_{A}^{\mu}=C E . B$ may provide a source for a spatial asymmetry of $n_{A}(x)$ along the direction of $B_{x}$. This is so, if one starts from a picture of the heavy-ion collision in which there is not only a strong $B_{x}$ field, but also a strong electric field $E$, and if the $E$ field is directed preferentially along (opposite) $B_{x}$ in the positive (negative) transverse half-plane $x>0(x<0)$, as one expects from general geometric considerations. In the study of Ref. [33], such a term was included in the dynamical evolution. As noted in the discussion following (6), if $E . B \neq 0$ during the hydrodynamic evolution, then the charge density flow along the direction of the magnetic field receives contributions from the Ohmic term in (6). In the present work, however, we restrict ourselves to quantifying the effects induced by the anomalous terms in the current.

We recall that the chiral magnetic wave leads to spatial asymmetries of the axial charge in response to a finite vector charge chemical potential, and it does not require a finite $E$ field. In contrast, a creation of spatial asymmetries in $n_{A}$ from $\partial_{\mu} j_{A}^{\mu}=C E . B$ requires a finite $E$ field but does not depend on a finite vector charge chemical potential. Here, we do not address the important question about the size of the spatial asymmetries in $n_{A}$ that may be created by this alternative mechanism, nor do we discuss the time scale on which such an asymmetry may arise in the collision or dynamical effects that arise outside a hydrodynamical framework. We note in this context that quark-gluon plasmas with finite $\mu_{A}$ are expected to be dynamically unstable. This plasma instability is not 


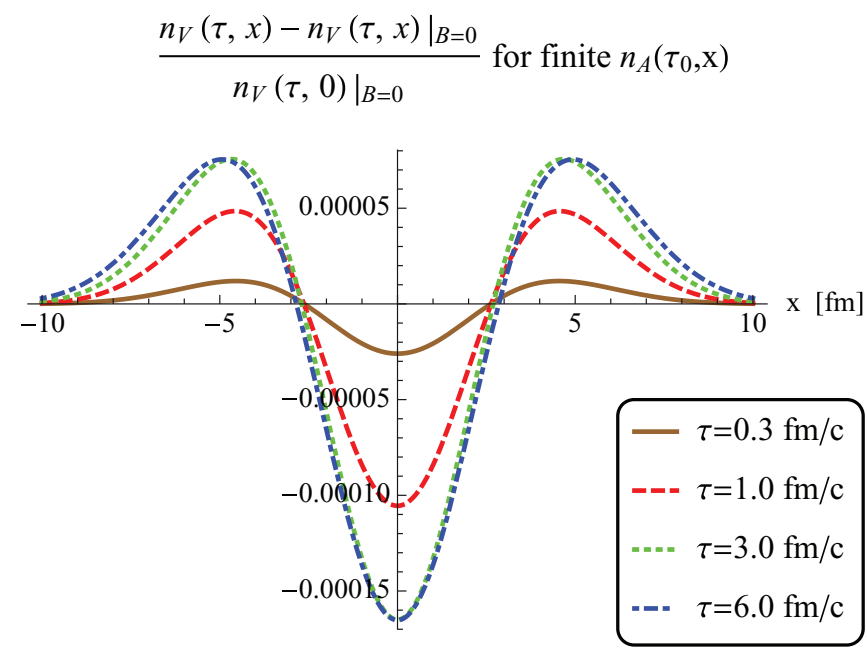

(a)

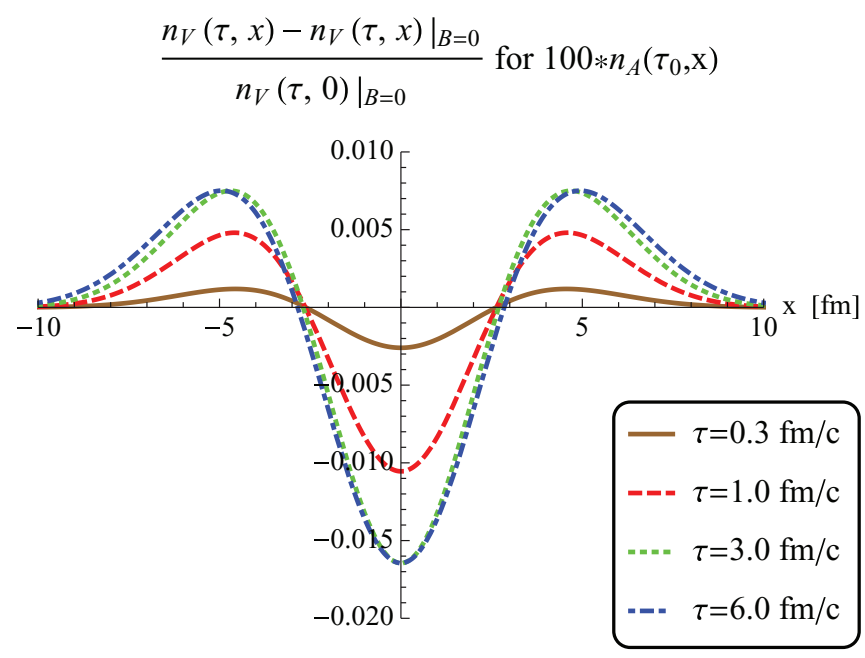

(b)

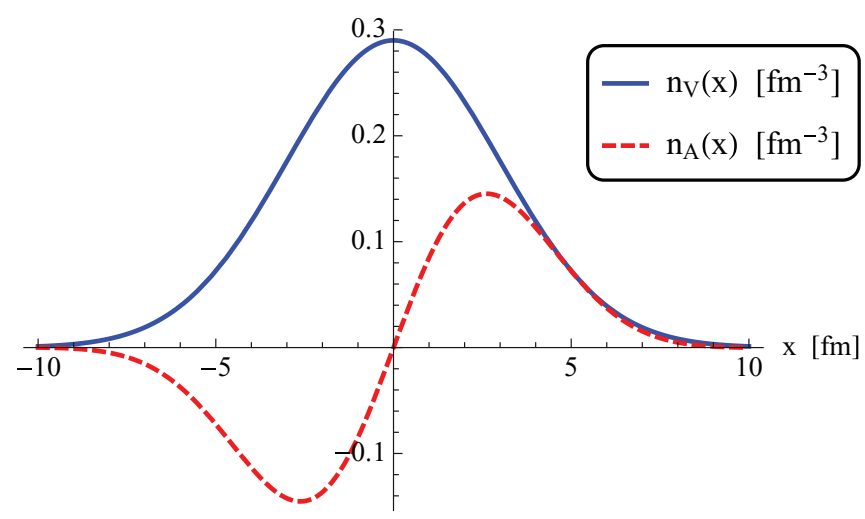

(c)

FIG. 6. (Color online) (a) and (b) Same as in Fig. 4(a), but calculated in (a) for the shown nonvanishing initial axial charge density distribution $n_{A}$ of (c), and calculated in (b) for a 100 times larger density $n_{A}$.

accounted for in anomalous hydrodynamics and it will tend to dampen $\mu_{A}$ by the exponential growth of color electromagnetic fields at the early stage of the collision [66] (see also [67,68]). By neglecting the role of this plasma instability, we will thus overestimate the importance of a finite axial charge density for the spatial separation of vector charges. This is the starting point of the present section: We simply accept the logical possibility that a finite spatial asymmetry of $n_{A}$ along $B_{x}$ may be present in the initial conditions and we seek an upper bound on the efficiency with which the CMW could transfer these spatial asymmetries in $n_{A}$ into a spatial separation of the vector charge distribution. To this end, we have repeated in Fig. 6 the calculation shown in the upper panel of Fig. 4 for the same input values and the same initial vector charge density $n_{V}\left(\tau_{0}, x\right)$, but for the nonvanishing axial charge density $n_{A}\left(\tau_{0}, x\right)$ shown in Fig. 6 . We find that-although the signal is still numerically small-it is enhanced by almost 5 orders of magnitude compared to the case in Fig. 4. This supports the general argument that the mechanism of vector charge separation from the chiral magnetic wave is significantly more efficient if a finite axial chemical potential needs not be generated dynamically but is present in the initial conditions. We further observe that for a given $x$ dependence of the initial asymmetry $n_{A}\left(\tau_{0}, x\right)$, the size of the vector charge separation grows approximately linearly with the amplitude of $n_{A}\left(\tau_{0}, x\right)$. In fact, rescaling $n_{A}$ in Fig. 6 by a factor 100 (which for the parameters chosen here still corresponds to $\mu_{A}(\tau, x) / T(\tau)<1$ everywhere), we find that the relative size of the normalized vector charge difference $\left(n_{V}-\left.n_{V}\right|_{B=0}\right) /\left.n_{V}\right|_{B=0}$ increases by a factor $\sim 100$ as well. We note as a speculative aside that if one starts from the numerical finding that the peak values of $e B_{x}$ reached in heavy-ion collisions are $O\left(\mathrm{~m}_{\pi}^{2}\right)$ and if one assumes that $|e E| \sim\left|e B_{x}\right| \sim O\left(m_{\pi}^{2}\right)$, then the nonconservation of the axial current is parametrically large, $\partial_{\mu} j_{A}^{\mu} \propto \frac{m_{\pi}^{4}}{e}$, and large initial values for $n_{A}$ may be feasible indeed.

\section{CONCLUSION}

The chiral magnetic wave is a direct and unavoidable consequence of formulating QCD hydrodynamics in an external electromagnetic field. Experimental confirmation of this phenomenon would be of considerable interest because it would underline the relevance of quantum anomalies for the properties of hot and dense matter. However, whether the chiral magnetic wave can lead to unambiguous signals of experimentally accessible strength in ultrarelativistic heavyion collisions (and whether the CMW can offer a dynamical explanation for experimental signals of given strength) is a question that requires theoretical exploration. In the present paper, we have shown that in an expanding fluid and for small charge densities, the chiral magnetic wave separates axial charges with a local velocity,

$$
v_{ \pm}(\tau)=\frac{C}{4 C_{1}} \frac{B_{x}(\tau)}{T^{2}(\tau)},
$$

where $C=\frac{e}{2 \pi^{2}}$ is the strength of the axial anomaly and $C_{1}$ is a factor of order $O(1)$ in the equation of state (25). For the special case of a fluid that satisfies to leading order in $\mu / T$ the symmetries of Bjorken's model and where propagating charges can be treated as perturbations at subleading order in $\mu / T$, we have derived explicitly the equations of motion for 
the chiral magnetic wave and the velocity (38). Moreover, we have argued on general grounds that we expect Eq. (38) to describe the CMW-induced velocity of charge separation also for fluids whose collective expansion differs from that of the Bjorken-type model studied in Secs. III and IV.

For choices of the magnetic field strength and of the timedependent temperature that are realistic for ultrarelativistic heavy-ion collisions, the values found for $v_{x}$ from (38) are nonrelativistic, and the resulting time-integrated value of the spatial separation of right- and left-handed charges tends to be very small; see Fig. 2. For the simplified Bjorken-type model studied in Sec. III, and for initial conditions without finite axial charge potential, the CMW therefore typically induces asymmetries in the electric charge distribution that we regard as being too small to be experimentally accessible because they affect on average the distribution of much less than one charge per collision. This conclusion is reached on the basis of a simplified model. While numerical results may change somewhat for the case of a fully realistic fluid dynamic simulation of relativistic heavy-ion collisions, we emphasize that our main conclusion is based on the logarithmic order of the asymmetry of charge separation $\left[O\left(10^{-8}\right)\right.$ in Fig. 4]-a more realistic simulation alone is unlikely to overcome this big suppression factor. As discussed in Sec. IV, however, there may be physics effects that lead to initial conditions with significant axial chemical potential so that phenomenological consequences can be much enhanced. The findings of Sec. IV, therefore, call for a better understanding of the initial conditions with which the CMW is initialized.

Note added in proof: One month after completion of our work, Ref. [69] appeared. This work states consistency with our findings, and it elaborates on a scenario according to which effects occurring at the freeze-out stage could give numerically significant contributions. Thus, enhanced phenomenological consequences of the CMW may arise not only from modified initial conditions, as argued in Sec. IV, but also from the hadronic decoupling at the end of the fluid dynamic evolution.

\section{ACKNOWLEDGMENTS}

One of us (S.F.T.) would like to thank the CERN TH Unit for hospitality during this work. We thank Navid Abbasi, Mohammad Ali-Akbari, Farhad Ardalan, Stefan Flörchinger, Eduardo Fraga, Massimo Giovannini, Dima Kharzeev, Larry McLerran, Misha Stephanov, Sergey Voloshin, and Ho-Ung Yee for useful discussions.
[1] G. E. Volovik, The Universe in a Helium Droplet (Oxford University Press, Oxford, 2003).

[2] G. E. Volovik and A. Vilenkin, Phys. Rev. D 62, 025014 (2000).

[3] M. Joyce and M. E. Shaposhnikov, Phys. Rev. Lett. 79, 1193 (1997).

[4] M. Giovannini and M. E. Shaposhnikov, Phys. Rev. D 57, 2186 (1998).

[5] A. Vilenkin, Phys. Rev. D 20, 1807 (1979).

[6] A. Vilenkin, Phys. Rev. D 21, 2260 (1980).

[7] A. Vilenkin, Phys. Rev. D 22, 3080 (1980).

[8] D. T. Son and P. Surówka, Phys. Rev. Lett. 103, 191601 (2009).

[9] J. Erdmenger, M. Haack, M. Kaminski, and A. Yarom, J. High Energy Phys. 01 (2009) 055.

[10] N. Banerjee, J. Bhattacharya, S. Bhattacharyya, S. Dutta, R. Loganayagam, and P. Surówka, J. High Energy Phys. 01 (2011) 094.

[11] K. Landsteiner, E. Megias, and F. Pena-Benitez, Phys. Rev. Lett. 107, 021601 (2011).

[12] K. Landsteiner, E. Megías, L. Melgar, and F. Pena-Benitez, J. High Energy Phys. 09 (2011) 121.

[13] Y. Neiman and Y. Oz, J. High Energy Phys. 03 (2011) 023.

[14] D. T. Son and N. Yamamoto, Phys. Rev. Lett. 109, 181602 (2012).

[15] M. A. Stephanov and Y. Yin, Phys. Rev. Lett. 109, 162001 (2012).

[16] D. T. Son and N. Yamamoto, Phys. Rev. D 87, 085016 (2013).

[17] J.-H. Gao, Z.-T. Liang, S. Pu, Q. Wang, and X.-N. Wang, Phys. Rev. Lett. 109, 232301 (2012).

[18] J.-W. Chen, S. Pu, Q. Wang, and X.-N. Wang, Phys. Rev. Lett. 110, 262301 (2013).

[19] D. E. Kharzeev and H.-U. Yee, Phys. Rev. D 84, 045025 (2011).

[20] A. V. Sadofyev and M. V. Isachenkov, Phys. Lett. B 697, 404 (2011).
[21] D. Kharzeev and A. Zhitnitsky, Nucl. Phys. A 797, 67 (2007).

[22] D. E. Kharzeev, L. D. McLerran, and H. J. Warringa, Nucl. Phys. A 803, 227 (2008).

[23] K. Fukushima, D. E. Kharzeev, and H. J. Warringa, Phys. Rev. D 78, 074033 (2008).

[24] A. Bzdak, V. Koch, and J. Liao, Phys. Rev. C 81, 031901 (2010).

[25] A. Bzdak, V. Koch, and J. Liao, Phys. Rev. C 83, 014905 (2011).

[26] S. Schlichting and S. Pratt, Phys. Rev. C 83, 014913 (2011).

[27] B. Keren-Zur and Y. Oz, J. High Energy Phys. 06 (2010) 006.

[28] D. E. Kharzeev and H.-U. Yee, Phys. Rev. D 83, 085007 (2011).

[29] D. E. Kharzeev and D. T. Son, Phys. Rev. Lett. 106, 062301 (2011).

[30] Y. Burnier, D. E. Kharzeev, J. Liao, and H.-U. Yee, Phys. Rev. Lett. 107, 052303 (2011).

[31] Y. Burnier, D. E. Kharzeev, J. Liao, and H.-U. Yee, arXiv:1208.2537.

[32] M. Stephanov and H.-U. Yee, Phys. Rev. C 88, 014908 (2013).

[33] M. Hongo, Y. Hirono, and T. Hirano, arXiv:1309.2823.

[34] S. A. Voloshin, Phys. Rev. C 70, 057901 (2004).

[35] I. V. Selyuzhenkov (STAR Collaboration), Rom. Rep. Phys. 58, 049 (2006)

[36] B. I. Abelev et al. (STAR Collaboration), Phys. Rev. Lett. 103, 251601 (2009).

[37] B. I. Abelev et al. (STAR Collaboration), Phys. Rev. C 81, 054908 (2010).

[38] B. Mohanty (STAR Collaboration), J. Phys. G 38, 124023 (2011).

[39] H. Ke (STAR Collaboration), J. Phys. Conf. Ser. 389, 012035 (2012).

[40] L. Adamczyk et al. (STAR Collaboration), Phys. Rev. Lett. 110, 142301 (2013).

[41] L. Adamczyk et al. (STAR Collaboration), Phys. Rev. C 89, 044908 (2014). 
[42] D. Adamova et al. (CERES Collaboration), Nucl. Phys. A 894, 41 (2012).

[43] W.-T. Deng and X.-G. Huang, Phys. Rev. C 85, 044907 (2012).

[44] Y. Neiman and Y. Oz, J. High Energy Phys. 09 (2011) 011.

[45] J. D. Bjorken, Phys. Rev. D 27, 140 (1983).

[46] S. S. Gubser, Phys. Rev. D 82, 085027 (2010).

[47] A. Muronga, Phys. Rev. C 69, 034903 (2004).

[48] C. R. Allton, M. Doring, S. Ejiri, S. J. Hands, O. Kaczmarek, F. Karsch, E. Laermann, and K. Redlich, Phys. Rev. D 71, 054508 (2005).

[49] S. S. Gubser, I. R. Klebanov, and A. W. Peet, Phys. Rev. D 54, 3915 (1996).

[50] T. Nishioka and T. Takayanagi, Phys. Rev. D 76, 044004 (2007).

[51] E. S. Fraga and A. J. Mizher, Phys. Rev. D 78, 025016 (2008).

[52] A. J. Mizher, M. N. Chernodub, and E. S. Fraga, Phys. Rev. D 82, 105016 (2010).

[53] G. S. Bali, F. Bruckmann, G. Endrodi, and A. Schafer, arXiv: 1310.8145 .

[54] S. Caron-Huot, P. Kovtun, G. D. Moore, A. Starinets, and L. G. Yaffe, J. High Energy Phys. 12 (2006) 015.

[55] D. Mateos and L. Patiño, J. High Energy Phys. 11 (2007) 025.

[56] H.-T. Ding, A. Francis, O. Kaczmarek, F. Karsch, E. Laermann, and W. Soeldner, Phys. Rev. D 83, 034504 (2011).
[57] P. K. Kovtun, D. T. Son, and A. O. Starinets, Phys. Rev. Lett. 94, 111601 (2005).

[58] K. Aamodt et al. (ALICE Collaboration), Phys. Rev. Lett. 105, 252301 (2010).

[59] B. B. Back, M. D. Baker, D. S. Barton, R. R. Betts, M. Ballintijn, A. A. Bickley, R. Bindel, A. Budzanowski et al., Phys. Rev. Lett. 91, 052303 (2003).

[60] V. Skokov, A. Y. Illarionov, and V. Toneev, Int. J. Mod. Phys. A 24, 5925 (2009).

[61] Y.-J. Mo, S.-Q. Feng, and Y.-F. Shi, Phys. Rev. C 88, 024901 (2013).

[62] V. Voronyuk, V. D. Toneev, W. Cassing, E. L. Bratkovskaya, V. P. Konchakovski, and S. A. Voloshin, Phys. Rev. C 83, 054911 (2011).

[63] A. Bzdak and V. Skokov, Phys. Lett. B 710, 171 (2012).

[64] K. Tuchin, Adv. High Energy Phys. 2013, 490495 (2013).

[65] L. McLerran and V. Skokov, arXiv:1305.0774.

[66] Y. Akamatsu and N. Yamamoto, Phys. Rev. Lett. 111, 052002 (2013).

[67] V. P. Kirilin, A. V. Sadofyev, and V. I. Zakharov, arXiv:1312.0895.

[68] Y. Akamatsu and N. Yamamoto, Phys. Rev. D 90, 125031 (2014).

[69] H.-U. Yee and Y. Yin, Phys. Rev. C 89, 044909 (2014). 